\title{
Modified Constrained Induced Movement Therapy Versus Mirror Therapy Analysis through Fugl-Meyer and Nine Hole Peg Scales
}

\section{Sneha Tiwari*}

Department of Neurology, India

*Corresponding Author: Sneha Tiwari, Department of Neurology, India.

Received: September17, 2019; Published: November 13, 2019

DOI: 10.31080/ASNE.2019.02.0123

\begin{abstract}
Background: To compare effectiveness of modified constrained induced movement therapy versus mirror therapy in improving hand dexterity in patients with sub-acute stroke.

Objective: To evaluate the effectiveness of modified constraint induced movement therapy (MCIMT) and Mirror therapy in improving hand dexterity to patients with sub-acute stroke.

Methods: 20 patients with sub-acute stroke were enrolled and divided into two groups: Modified constraint induced movement therapy (MCIMT) and mirror therapy (MT). Training for MCIMT will be for 2 hrs per day, 5 days per week for 5 weeks and training for mirror therapy will be 2 hrs per day, 5 days per week for 5 weeks with the help of assessment scales Fugl-meyer scale and Nine hole peg scale.

Keywords: Modified Constraint Induced Movement Therapy (MCIMT); Mirror Therapy (MT)
\end{abstract}

Experiment sample description for statistical analysis

Sample Size is 20 patients which is divided in to 2 groups of 10 sample size each randomly.

Male vs female ratio in the sample

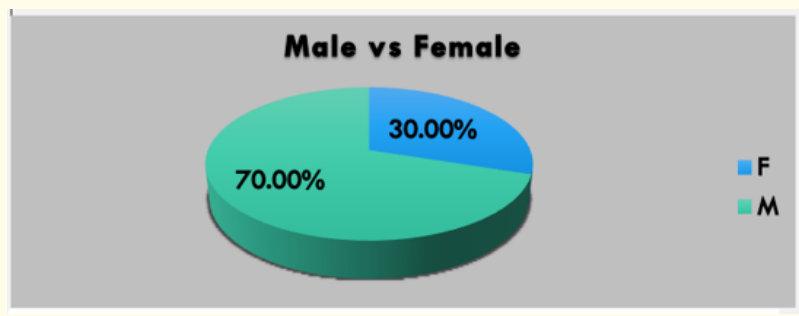

Figure 1

$70 \%$ of the sample were the males and $30 \%$ were the female in the sample.
Stroke type ratio in the sample

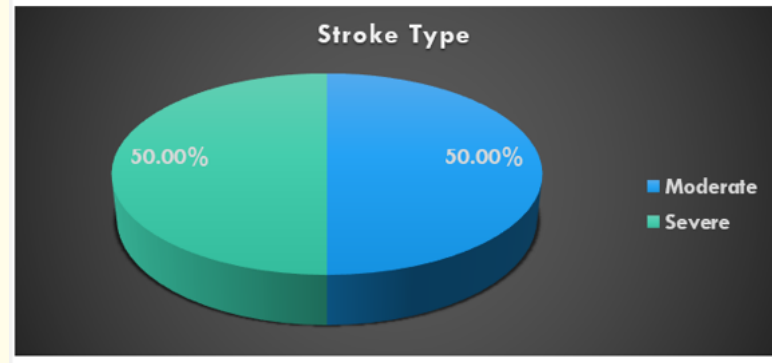

Figure 2

$50 \%$ were hemorrhagic and $50 \%$ were Ischemic were the stroke type patients in the sample. 
Stroke severity type in the sample

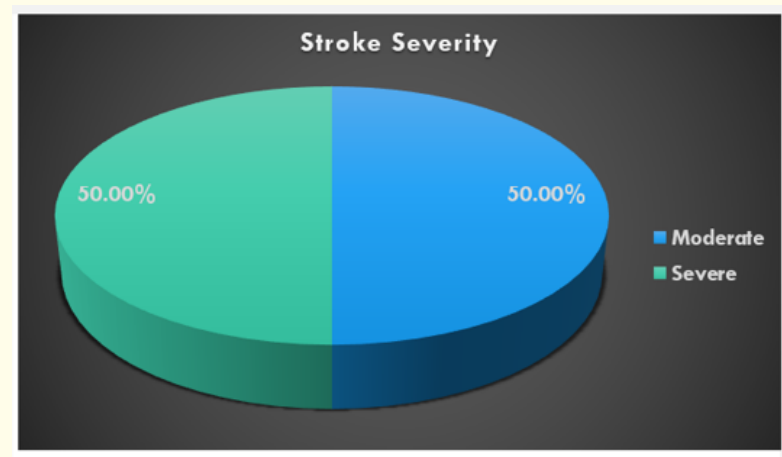

Figure 3

50\% were Moderate and 50\% were Severe were the stroke type patients in the sample.

Time since stroke in the sample

More than 3 months cases were taken for the study in the sample.

Age of the patients in the sample

More than 40 years of age were considered for the study in the sample.

\section{Data collection and experimental conditions}

\section{Inclusion criteria}

1. $40-60$ years of age.

2. Men and women with sub-acute stroke.

3. Subjects with dominant side hemi-paretic.

4. Brunstromm's stage (3 - 5 level).

5. Mini-Mental Status Examination. (MMSE) not less than 24 score.

\section{Exclusion criteria}

1. Acute and Chronic.

2. Subjects more than 60 years old.

3. Subjects less than 40years old.

4. Patients with visual deficits.

5. Severe cognitive impairment.

\section{Study variables}

- $\quad$ Constrain induced movement therapy.

- $\quad$ Mirror therapy.
Equipments and materials

1. Nine-holepegboard.

2. Stopwatch.

3. Mirror 75 x 64 centimeters.

\section{Statistical tools}

1. Computer.

2. The Excel Statistical Software version is used with the help of statistic after collection of data (pre-intervention and post intervention data).

\section{Assessment tools}

1. Nine-hole peg scale: It is simple and easy, simple and quick to use, to assess fine motor coordination and handeye coordination. In this scale first unaffected (non-dominant hand) is practiced, followed by affected/dominant hand. It has high inter-rater reliability and evidence for concurrent and convergent validity.

2. Fugl-Meyer scale: It is widely used to assess and measure fine motor activities. Hand activity is assessed with eight components, this is made by assistance provided to the arm at the elbow and just proximal to the wrist in order to position the arm for the grasp tasks. I.e. 8a, finger mass flexion, 8b, finger mass extension. 8c, extension of MCP $2^{\text {nd }}$ to $5^{\text {th }}$ fingers and flexion of proximal and distal IP joints are assumed patient holding a brief case, $8 \mathrm{~d}$ grasping with involving thumb abduction and first digit holding scrap of a paper. 8e, it involves holding a pen or a pencil involving thumb and index finger prehension. 8f, grasping of small cylindrical things like a small can. Opposing the volar surface of the thumb and fingers. 8g, spherical grasping with the help of a tennis ball.

\section{Data-collection procedure}

The patient with sub-acute stroke will be referred to college of physiotherapy, the subject will be checked for inclusion and exclusion criteria. If inclusion criteria is met, they will be asked to fill the consent form, If they are willing to participate in the study. Then the subjects will be included in the study and divided into two groups by simple random sampling method.

This will be followed by a pre-Intervention data collection which is done by mini-mental status examination (MMSE). 
The MMSE is the best tool that can be used to systematically and thoroughly assess mental status. It is an 11-questionable measure that tests five areas of cognitive function: Orientation, registration, attention and calculation, recall and language. The maximum score is 30 . A score of 23 or lower is indicative of cognitive impairment. This test takes only 5 - 10 minutes to administer and therefore, it is practical to use repeatedly and routinely.

Intervention

Subjects are divided into 2 groups. Group A are treated with constrained induced movement therapy and Group B treated with mirror therapy.

Active assisted exercises and active range of motion (AROM) exercises are done at the end of the treatment to whole upper limb to both the groups.

Both the groups are taught DOS and Don'ts.

Group A

Subjects who are assigned into this group will be treated with constrained induced movement therapy, and AROM exercises.

Group B

Subjects who are assigned into this group will be treated with mirror therapy, activity of daily living (ADL) exercises and AROM exercises.

MCIMT will be for 2 hrs per day, 5 days per week for 5 weeks and training for mirror therapy will be 2 hrs per day, 5 days per week for 5 weeks with the help of assessment scales Fugl-meyer scale and Nine hole peg scale.

Fugl-meyer scale test

Method used to perform statistical analysis on the sample data.

We have used the T-Test Unpaired test sample assuming unequal variances test for 2 reasons:

1. The sample size is less than 30 .

2. Sample standard deviations are unknown.

So, we are using T -Test for Unpaired samples when their Variances are Unknown.

Modified constraint induced movement therapy (MCIMT) and mirror therapy (MT) Test on fugl-meyer scale test

Fugl-meyer scale

- For No score- 0

- $\quad$ For medium score- 1

- $\quad$ For best score- 2
Data- Fugl-meyer scale

\begin{tabular}{|l|c|c|c|}
\hline Patient No. & MCIMT-FMS & Patient No. & Mirror-FMS \\
\hline Patient 1 & 43 & Patient 11 & 40 \\
\hline Patient 2 & 37 & Patient 12 & 40 \\
\hline Patient 3 & 44 & Patient 13 & 43 \\
\hline Patient 4 & 41 & Patient 14 & 40 \\
\hline Patient 5 & 39 & Patient 15 & 38 \\
\hline Patient 6 & 34 & Patient 16 & 39 \\
\hline Patient 7 & 36 & Patient 17 & 39 \\
\hline Patient 8 & 44 & Patient 18 & 44 \\
\hline Patient 9 & 38 & Patient 19 & 42 \\
\hline Patient 10 & 32 & Patient 20 & 42 \\
\hline
\end{tabular}

Table 1: Data input of combined "MCIM and Mirror".

Hypothesis- Fugl-Meyer Scale

- $\quad$ Null Hypothesis: Mean score of MCIMT-FMS are Equal to Mean Score of Mirror-FMS.

- $\quad$ Alternative Hypothesis: Mean score of MCIMT-FMS are not equal to Mean Score of Mirror-FMS.

Test Results: Fugl-Meyer Scale

\begin{tabular}{|l|c|c|}
\hline & MCIMT-FMS & Mirror-FMS \\
\hline Mean & 36.1 & 40.7 \\
\hline Variance & 9.655555556 & 3.788888889 \\
\hline Observations & 10 & 10 \\
\hline Hypothesized Mean Difference & 0 & \\
\hline Df & 15 & \\
\hline t Stat & -3.967221065 & \\
\hline P(T<=t) one-tail & 0.000619643 & \\
\hline t Critical one-tail & 1.753050356 & \\
\hline P(T<=t) two-tail & 0.001239285 & \\
\hline t Critical two-tail & 2.131449546 & \\
\hline
\end{tabular}

Table 2: Results of the T-test of MCIMT over mirror therapy.

Analysis- Fugl-Meyer Scale

Benchmark P Value for one tail test is .05 and Benchmark P-Value for two tail tests is .025.

P Value one tail is .000619 which is less than .05 and P-Value two tail is .00123 which is less than .025 , suggests there is sufficient evidence to believe that the performance of Modified Constraint Induced Movement Therapy (MCIMT) and Mirror Therapy (MT) conducted through Fugl-meyer test are not same, here in this experiment the Mirror Test are showing better results over Modified Constraint Induced Movement Therapy (MCIMT). 
Combined modified constraint induced movement therapy (MCIMT) and mirror therapy (MT) test to mirror therapy FMS test

Data- Fugl-Meyer Scale

\begin{tabular}{|l|c|c|}
\hline Patient No. & MCIMT & MCIMT + Mirror \\
\hline Patient 1 & 42 & 46 \\
\hline Patient 2 & 35 & 43 \\
\hline Patient 3 & 39 & 44 \\
\hline Patient 4 & 36 & 42 \\
\hline Patient 5 & 37 & 42 \\
\hline Patient 6 & 33 & 43 \\
\hline Patient 7 & 34 & 44 \\
\hline Patient 8 & 37 & 42 \\
\hline Patient 9 & 37 & 39 \\
\hline Patient 10 & 31 & 39 \\
\hline
\end{tabular}

Table 3: Data input of combined "MCIMT+Mirror and MCIMT". Hypothesis- Fugl-Meyer Scale

- $\quad$ Null Hypothesis: Mean score of Combined MCIMT and Mirror are Equal to Mean Score of MCIMT.

- Alternative Hypothesis: Mean score of Combined MCIMT and Mirror are Not Equal to Mean Score of MCIMT.

We are using T-Test for Paired Samples as a test of hypothesis.

Test Results: Fugl-Meyer Scale

\begin{tabular}{|l|c|c|}
\hline & MCIMT & $\begin{array}{c}\text { MCIMT + } \\
\text { Mirror }\end{array}$ \\
\hline Mean & 36.1 & 42.4 \\
\hline Variance & 9.655555556 & 4.711111111 \\
\hline Observations & 10 & 10 \\
\hline Pearson Correlation & 0.553536752 & \\
\hline Hypothesized Mean Difference & 0 & \\
\hline Df & 9 & \\
\hline t Stat & -7.584308744 & \\
\hline P(T<=t) one-tail & $1.69069 \mathrm{E}-05$ & \\
\hline t Critical one-tail & 1.833112933 & \\
\hline P $(\mathrm{T}<=t)$ two-tail & $3.38138 \mathrm{E}-05$ & \\
\hline t Critical two-tail & 2.262157163 & \\
\hline
\end{tabular}

Table 4: Results of the T-test of MCIMT+Mirror over MCIMT.

\section{Analysis- Fugl-Meyer Scale}

Benchmark P Value for one tail test is .05 and Benchmark P-Value for two tail tests is .025 .

P Value one tail is .0000169 which is less than .05 and P-Value two tail is .0000338 which is less than .025 , suggests there is sufficient evidence to believe that the performance of Combined impact of the Modified Constraint Induced Movement Therapy (MCIMT) and Mirror Therapy (MT) over Modified Constraint Induced Movement Therapy (MCIMT) conducted through Fugl-meyer test are not same, here in this experiment the Combined Modified Constraint Induced Movement Therapy (MCIMT) is performing better than the MCIMT test as per the table 4 results.

Combined mirror therapy and modified constraint induced movement therapy (MCIMT) to mirror therapy FMS test

Data- Fugl-Meyer Scale

\begin{tabular}{|l|c|c|}
\hline Patient No. & Mirror-FMS & Mirror + MCIMT \\
\hline Patient 11 & 40 & 49 \\
\hline Patient 12 & 40 & 49 \\
\hline Patient 13 & 43 & 43 \\
\hline Patient 14 & 40 & 46 \\
\hline Patient 15 & 38 & 38 \\
\hline Patient 16 & 39 & 38 \\
\hline Patient 17 & 39 & 43 \\
\hline Patient 18 & 44 & 49 \\
\hline Patient 19 & 42 & 50 \\
\hline Patient 20 & 42 & 49 \\
\hline
\end{tabular}

Table 5: Data input of combined "Mirror+MCIMT and Mirror". Hypothesis- Fugl-Meyer Scale

- Null Hypothesis: Mean score of Combined Mirror and MCIMT are Equal to Mean Score of Mirror.

- Alternative Hypothesis: Mean score of Combined Mirror and MCIMT are Not Equal to Mean Score of Mirror.

We are using T-Test for paired samples as a test of hypothesis. 
Test Results: Fugl-Meyer Scale

\begin{tabular}{|l|c|c|}
\hline & Mirror-FMS & $\begin{array}{c}\text { Mirror + } \\
\text { MCIMT }\end{array}$ \\
\hline Mean & 40.7 & 45.4 \\
\hline Variance & 3.788888889 & 21.6 \\
\hline Observations & 10 & 10 \\
\hline Pearson Correlation & 0.592000016 & \\
\hline Hypothesized Mean Difference & 0 & \\
\hline Df & 9 & \\
\hline t Stat & -3.87942807 & \\
\hline P(T<=t) one-tail & 0.00186733 & \\
\hline t Critical one-tail & 1.833112933 & \\
\hline P(T<=t) two-tail & 0.003734659 & \\
\hline$t$ Critical two-tail & 2.262157163 & \\
\hline
\end{tabular}

Table 6: Results of the T-test of Mirror + MCIMT over mirror.

Analysis- Fugl-Meyer Scale

Benchmark P Value for one tail test is .05 and Benchmark P-Value for two tail tests is .025 .

P Value one tail is .0018 which is less than .05 and P-Value two tail is .0037 which is less than .025 , suggests there is sufficient evidence to believe that the performance of Combined impact of the Mirror Therapy and Modified Constraint Induced Movement Therapy (MCIMT) over Mirror Therapy test conducted through Fugl-meyer test are not same, here in this experiment the Combined Mirror Therapy and Modified Constraint Induced Movement Therapy (MCIMT) is performing better than the Mirror Therapy as per the table 6 results.

Nine hole peg test

Modified constraint induced movement therapy (MCIMT) and mirror therapy (MT) test on nine hole peg test

Nine hole peg scale

- $\quad$ Time to fill the Nine holes from the right hand = Average of 2 trails.

- $\quad$ Time to fill the Nine holes from the left hand = Average of 2 trails.

Average of the 4 trails is the score which we have taken for the T-Test.
Data- NHPS

\begin{tabular}{|l|c|c|c|}
\hline Patient No. & MCIMT - NHPS & Patient No. & Mirror -NHPS \\
\hline Patient 1 & 16.65 & Patient 11 & 15.87 \\
\hline Patient 2 & 16.89 & Patient 12 & 15.41 \\
\hline Patient 3 & 18.34 & Patient 13 & 16.39 \\
\hline Patient 4 & 17.71 & Patient 14 & 15.23 \\
\hline Patient 5 & 16.46 & Patient 15 & 16 \\
\hline Patient 6 & 17.34 & Patient 16 & 15.81 \\
\hline Patient 7 & 16.86 & Patient 17 & 15.37 \\
\hline Patient 8 & 16.94 & Patient 18 & 15.89 \\
\hline Patient 9 & 16.9 & Patient 19 & 15.75 \\
\hline Patient 10 & 16.92 & Patient 20 & 15.84 \\
\hline
\end{tabular}

Table 7: Data input of combined "MCIM and Mirror".

Hypothesis- NHPS

- $\quad$ Null Hypothesis: Mean score of MCIMT-NHPS are Equal to Mean Score of Mirror-NHPS.

- Alternative Hypothesis: Mean score of MCIMT-NHPS are not equal to Mean Score of Mirror-NHPS.

We are using T-Test for Unpaired Samples with Unequal Variances.

Test Results: NHPS

\begin{tabular}{|l|c|c|}
\hline & MCIMT & Mirror \\
\hline Mean & 17.101 & 15.756 \\
\hline Variance & 0.30878778 & 0.11642667 \\
\hline Observations & 10 & 10 \\
\hline Hypothesized Mean Difference & 0 & \\
\hline df & 15 & \\
\hline t Stat & 6.52256296 & \\
\hline $\mathrm{P}(\mathrm{T}<=\mathrm{t})$ one-tail & $4.8226 \mathrm{E}-06$ & \\
\hline $\mathrm{t}$ Critical one-tail & 1.75305036 & \\
\hline $\mathrm{P}(\mathrm{T}<=\mathrm{t})$ two-tail & $9.6451 \mathrm{E}-06$ & \\
\hline $\mathrm{t}$ Critical two-tail & 2.13144955 & \\
\hline
\end{tabular}

Table 8: Results of the T-Test of MCIMT over mirror therapy.

Analysis- NHPS

Benchmark P Value for one tail test is .05 and Benchmark P-Value for two tail tests is .025 .

P Value one tail is .00000482 which is less than .05 and P-Value two tail is .000009645 which is less than .025 , suggests there is sufficient evidence to believe that the performance of Modified Con- 
straint Induced Movement Therapy (MCIMT) and Mirror Therapy (MT) conducted through Nine Hole Peg test are not same, here in this experiment the Mirror Test are showing better results over Modified Constraint Induced Movement Therapy (MCIMT) as per table 8.

Combined modified constraint induced movement therapy (MCIMT) and mirror therapy (MT) test to mirror therapy nine hole peg test

Data- NHPS

\begin{tabular}{|l|c|c|}
\hline Patient No. & MCIMT & MCIMT + Mirror \\
\hline Patient 1 & 16.65 & 14.28 \\
\hline Patient 2 & 16.92 & 14.88 \\
\hline Patient 3 & 16.89 & 14.46 \\
\hline Patient 4 & 18.34 & 14.53 \\
\hline Patient 5 & 17.71 & 14.68 \\
\hline Patient 6 & 16.46 & 14.6 \\
\hline Patient 7 & 17.34 & 14.57 \\
\hline Patient 8 & 16.86 & 14.9 \\
\hline Patient 9 & 16.94 & 14.66 \\
\hline Patient 10 & 16.9 & 14.47 \\
\hline
\end{tabular}

Table 9: Data input of combined "MCIMT+Mirror and MCIMT".

\section{Hypothesis- MCIMT+MIRROR TO MIRROR (NHPS)}

- Null Hypothesis: Mean score of Combined MCIMT and Mirror are Equal to Mean Score of MCIMT (NHPS).

- Alternative Hypothesis: Mean score of Combined MCIMT and Mirror are Not Equal to Mean Score of MCIMT (NHPS).

We are using T-Test for Paired Samples as a test of hypothesis.

Test Results: MCIMT+Mirror to Mirror (NHPS)

\begin{tabular}{|l|c|c|}
\hline & MCIMT & MCIMT+Mirror \\
\hline Mean & 17.101 & 14.603 \\
\hline Variance & 0.308787778 & 0.03589 \\
\hline Observations & 10 & 10 \\
\hline Pearson Correlation & 0.023399491 & \\
\hline $\begin{array}{l}\text { Hypothesized Mean } \\
\text { Difference }\end{array}$ & 0 & \\
\hline Df & 9 & \\
\hline$t$ Stat & 13.55226831 & \\
\hline $\mathrm{P}(\mathrm{T}<=\mathrm{t})$ one-tail & $1.3574 \mathrm{E}-07$ & \\
\hline $\mathrm{t}$ Critical one-tail & 1.833112933 & \\
\hline $\mathrm{P}(\mathrm{T}<=\mathrm{t})$ two-tail & $2.71481 \mathrm{E}-07$ & \\
\hline $\mathrm{t}$ Critical two-tail & 2.262157163 & \\
\hline
\end{tabular}

Table 10: Results of the T-test of MCIMT+Mirror over MCIMT.

\section{Analysis- NHPS}

Benchmark P Value for one tail test is .05 and Benchmark P-Value for two tail tests is .025 .

P Value one tail is .000000135 which is less than .05 and P-Value two tail is .000000271 which is less than .025 , suggests there is sufficient evidence to believe that the performance of Combined impact of the Modified Constraint Induced Movement Therapy (MCIMT) and Mirror Therapy (MT) over Modified Constraint Induced Movement Therapy (MCIMT) conducted through Nine Hole Peg test are not same, here in this experiment the Combined Modified Constraint Induced Movement Therapy (MCIMT) is performing better than the MCIMT test as per the table 10 results.

Average time taken to fill the Nine Hole Peg in case of patients who have received MCIMT and Mirror therapy have taken 14.603 seconds which is lesser than as patients who have taken only MCIMT therapy with their average time to fill the nine hole is 17.1 seconds.

Combined mirror therapy and modified constraint induced movement therapy (MCIMT) to mirror therapy nine hole peg test

\section{Data- NHPS}

\begin{tabular}{|l|c|c|}
\hline Patient No. & Mirror-NHPS & Mirror + MCIMT -NHPS \\
\hline Patient 11 & 15.87 & 14.87 \\
\hline Patient 12 & 15.84 & 14.9 \\
\hline Patient 13 & 15.41 & 15.03 \\
\hline Patient 14 & 16.39 & 15 \\
\hline Patient 15 & 15.23 & 14.76 \\
\hline Patient 16 & 16 & 14.36 \\
\hline Patient 17 & 15.81 & 14.88 \\
\hline Patient 18 & 15.37 & 14.48 \\
\hline Patient 19 & 15.89 & 14.76 \\
\hline Patient 20 & 15.75 & 14.92 \\
\hline
\end{tabular}

Table 11: Data input of combined "Mirror+MCIMT and Mirror". Hypothesis- NHPS

- Null Hypothesis: Mean score of Combined Mirror and MCIMT are Equal to Mean Score of Mirror.

- Alternative Hypothesis: Mean score of Combined Mirror and MCIMT are Not Equal to Mean Score of Mirror.

We are using T-Test for paired samples as a test of hypothesis. 
Test Results: NHPS

\begin{tabular}{|l|c|c|}
\hline & Mirror & Mirror+MCIMT \\
\hline Mean & 15.756 & 14.796 \\
\hline Variance & 0.11642667 & 0.047515556 \\
\hline Observations & 10 & 10 \\
\hline Pearson Correlation & 0.14795307 & \\
\hline $\begin{array}{l}\text { Hypothesized Mean Differ- } \\
\text { ence }\end{array}$ & 0 & \\
\hline Df & 9 & \\
\hline t Stat & 8.05803282 & \\
\hline $\mathrm{P}(\mathrm{T}<=\mathrm{t})$ one-tail & $1.0446 \mathrm{E}-05$ & \\
\hline $\mathrm{t}$ Critical one-tail & 1.83311293 & \\
\hline $\mathrm{P}(\mathrm{T}<=\mathrm{t})$ two-tail & $2.0892 \mathrm{E}-05$ & \\
\hline $\mathrm{t}$ Critical two-tail & 2.26215716 & \\
\hline
\end{tabular}

Table 12: Results of the T-test of Mirror+MCIMT over mirror.

\section{Analysis- NHPS}

Benchmark P Value for one tail test is .05 and Benchmark P-Value for two tail tests is .025 .

P Value one tail is .0018 which is less than .05 and P-Value two tail is .0037 which is less than .025 , suggests there is sufficient evidence to believe that the performance of Combined impact of the Mirror Therapy and Modified Constraint Induced Movement Therapy (MCIMT) over Mirror Therapy test conducted through Nine Hole Peg Test are not same, here in this experiment the Combined Mirror Therapy and Modified Constraint Induced Movement Therapy (MCIMT) is performing better than the Mirror Therapy as per the table 12 results.

Time taken by Patients who have taken Mirror and MCIMT is 14.796 seconds as compared to patients who have taken mere Mirror Therapy is 15.756 which is higher than patients who have taken the Mirror as well as MCIMT Therapy both.

Test-retest reliability and inter-rater reliability test report for nine hole peg test

\begin{tabular}{|c|c|c|}
\hline & $\begin{array}{c}\text { Test-Retest } \\
\text { Reliability }\end{array}$ & Interrater reliability \\
\hline MCIMT & $90 \%$ & $88 \%$ \\
\hline Mirror & $95 \%$ & $94 \%$ \\
\hline MCIMT+Mirror & $98 \%$ & $94 \%$ \\
\hline Mirror + MCIMT & $99 \%$ & $97 \%$ \\
\hline
\end{tabular}

Table 13

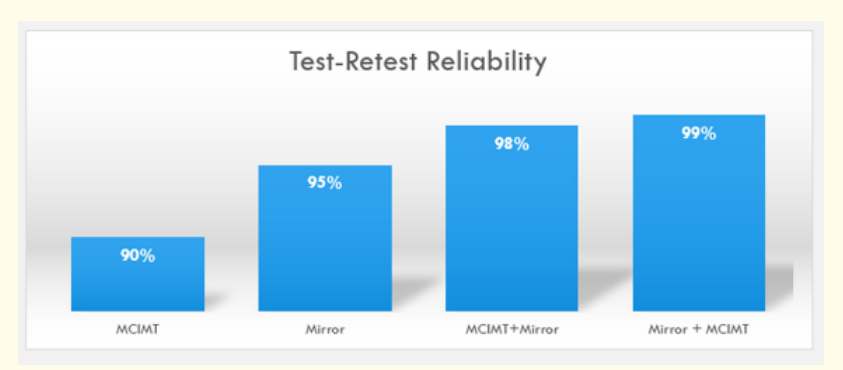

Figure 4

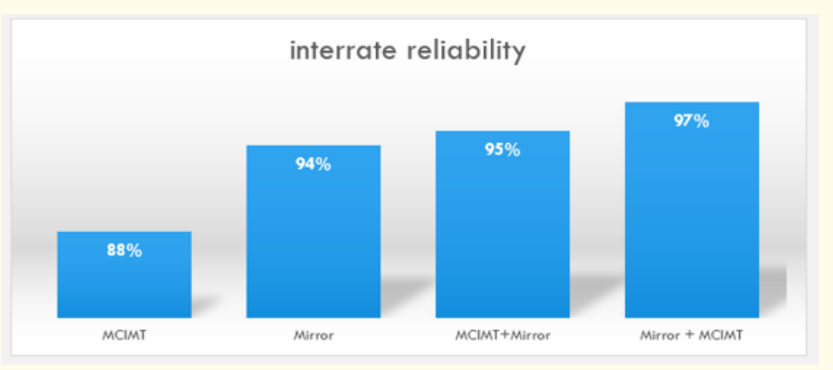

Figure 5

"MCIMT+Mirror" and "Mirror+MCIMT" combined therapies are showing better Test-Re-Test Reliabilities than MCIMT alone and Mirror alone therapies.

"MCIMT+Mirror" and "Mirror+MCIMT" combined therapies are showing better Inter-rater Reliabilities than MCIMT alone and Mirror alone therapies.

\section{Conclusion}

The Modified constraint induced movement therapy (MCIMT) combined with mirror therapy (MT) showed more improvement compared to MCIMT only group in the improvement of functions of hand dexterity for the patients with sub-acute stroke.

\section{Bibliography}

Snehalata Tiwari, Postgraduate. I am first year masters student studying Neurology. My research Effectiveness of the modified constraint induced movement therapy (MCIMT) versus mirror therapy in improving hand dexterity in patients with sub-acute stroke mainly focuses on improving the fine movements of the patients which are used in ADL's and other motor functions with increase in motivational and functional activities of the patients.

\section{Volume 2 Issue 12 December 2019 \\ (C) All rights are reserved by Sneha Tiwari.}

